\title{
Unilateral multiple Renal Congenital Anomalies: Ectopia; Double Collecting system; uretero-pelvic junction obstruction; uretero- vesical junction obstruction;Lower Moiety ureter to Upper Moiety Ureter Reflux; A Case Report
}

\author{
Seyed Mohammadreza Rabani ${ }^{1}$ \\ ${ }^{1}$ Yasuj University of Medical Sciences
}

March 17, 2021

\begin{abstract}
Urinary tract is a site for common anomalies both in upper and lower tracts, but gathering all of the anomalies reported in our patient and also being unilaterally, may be a rare entity. Hereby we report a case with such a rare condition.
\end{abstract}

\section{Title page}

Title: Unilateral multiple Renal Congenital Anomalies: Ectopia, Double Collecting system, Uretero- pelvic Junction Obstruction, Uretero-Vesical Junction Obstruction, Lower Moiety Ureter to Upper Moiety Ureter Reflux; A Case Report

\section{authors name and Affiliation:}

Seyed Mohammadreza Rabani; Associate Professor of Urology and Renal TX

Beheshti Teaching Hospital; Yasuj University of Medical Sciences; Yusuj; Iran

smrrabani@gmail.com; +989177411389

\section{The Corresponding Author:}

Seyed Mohammadreza Rabani; Associate Professor of Urology and Renal TX

Beheshti Teaching Hospital; Yasuj University of Medical Sciences; Yusuj; Iran

smrrabani@gmail.com; +989177411389, fax no: +987433235153

Finally, I have no relation to the lists or from North Korea, Crimea and comply with at least one of the conditions (2)(a), 2(b)(i) or 2(b)(ii), and I don't have any relation to other governments that are under international sanctions.

Key Clinical Message

Although the urinary tract is a site for common anomalies, but gathering all of the anomalies such as UPJO, UVJO, Ectopia, double collecting system, and reflex of lower moiety to upper one, reported in our patient, may be rare congenital anomalies

Unilateral multiple Renal Congenital Anomalies: Ectopia; Double Collecting system; uretero-pelvic junction obstruction; uretero- vesical junction obstruction, Lower Moiety Ureter to Upper Moiety Ureter Reflux; A Case Report 


\section{Abstract}

Urinary tract is a site for common anomalies, but gathering all of the anomalies reported in our patient, and also being unilaterally, may be a rare entity. Hereby we report a case with such a rare condition.

Key words: Renal congenital anomalies, renal ectopia, congenital anomalies of the kidneys, urinary tract system

\section{Introduction}

Congenital anomalies of the urinary tract have a wide spectrum including upper and lower urinary tract anomalies such as renal hypoplasia, dysplasia, aplasia, ectopia, supernumerary, duplication, uretero-pelvic junction obstruction (UPJO), uretero-vesical junction obstruction (UVJO), vesico-ureteral reflux (VUR), ureterocele, posterior urethral valve, bladder exstrophy, and many other anomalies. The causes for these wide ranges of urinary tract anomalies are related to abnormal embryologic development of the urinary tract $(1,2)$. The urinary tract system is substantially derived from the intermediate mesoderm $(3,4)$, and base on the underlying defect and the disturbances in the developmental process, different types of congenital anomalies may develop in the urinary system.

Case Presentation

A 19-year-old army male presented with left lower quadrant (LLQ) abdominal pain after a blunt abdominal trauma, accompanied with gross hematuria. He was healthy and asymptomatic until that during a sport activity has a blunt abdominal trauma and developed pain and gross hematuria. On arrival to emergency room, physical examination revealed LLQ tenderness with stable vital signs. Primary laboratory data showed normal renal function tests with gross hematuria. Ultrasonography suggested ectopic pelvic left Kidney with hydronephrosis and UPJO with distal ureteral dilatation. Intravenous pyelography (Figure 1) confirmed the same findings. Renal DTPA scanning (Figure 2) was done that suggested ectopia, double collecting system, lower moiety uretero-pelvic junction obstruction and a common distal ureter with UVJO. Cystoscopy and try to perform ureteroscopy were failed to pass a catheter into the left ureter that was a plan for retrograde uretero-pyelography, because the UVJ area was very narrow. Exploration was done through a Gibson's retroperitoneal incision. The upper moiety ureter was significantly dilated, but the lower moiety was thin and narrow, both of them were ended into a tapered segment typical for adynamic segment and UVJO. Pyeloplasty and ureteroneocystostomy were carried out simultaneously. 3 months and 6 months Follow-up visits revealed no relevant complaint and the patient was asymptomatic, but denied any study for Follow-up due to COVID 19 situation. The patient has an uneventful post-operative 18 months follow up.

\section{Discussion}

Development of the urinary tract is a very complex process and depends on regular and precise events. Normal Development of the urinary system also needs integration of a variety of cell populations that have different embryologic origins (1-3). The final products of these events are the functional and the conduit part of the urinary system. The evolution of our knowledge in genetics and molecular biology has greatly advanced our understanding about pathophysiology of urinary tract congenital anomalies $(\mathbf{5 , 6})$. Although anomalies of the urinary tract are among the most common anomalies of the body, there are also a great number of varieties of anomalies in upper and lower urinary tract system.

In this patient we have accidentally encountered Unilateral multiple Renal Congenital Anomalies: Ectopia; Double Collecting system; UPJO, and UVJO. Each of these has its own pathophysiology and clinical presentation that will be discussed briefly.

\section{Renal Ectopia}

An ectopic kidney is a kidney that isn't placed in its normal flank fossa position. It has 2 kinds: cross ectopia in which one of the kidneys crosses the midline and both kidneys are located in one side of the body, and simple ectopia in which the kidney may be ipsilaterally located thoracic, cephalad, lower abdominal, or in the pelvis like our patient. The cause of this anomaly may be a defect in ascent process of the kidney or 
abnormal ureteric bud. An ectopic kidney may be asymptomatic lifelong, or may be associated with other abnormalities such as UPJO, because of high inserted ureter, malrotated kidney, and aberrant vessels.

Duplicated System

Duplicated system may be complete or partial. It is the result of unusual development of ureteral bud. In complete duplication, two ureteral buds emerge from the wolfian duct and results in two complete sets of kidneys and ureters that may insert into the bladder separately. Partial duplication can be a result of ureteral bud stalk bifurcation before invading or prior to initial branching of the primary ureteral bud into the metanephric mesenchyme (7). In complete duplicated system, the Wiegert-Meyer rule can be seen in $95 \%$ of the cases with consequences of vesico-ureteral reflux and ureterocle or ectopic ureter, but in our case UVJO was seen. Duplication system is more common in women than in men with a ratio of 2:1 (7).

Ureteropelvic junction obstruction (UPJO)

UPJO is an obstructive uropathy at the level of junction between renal pelvis and the origin of the ureter and may be intrinsic or extrinsic in nature. It accounts for about $50 \%$ of all intrauterine detectable hydronephrosis and it is really the most common cause of congenital obstructive nephropathy $(8,9)$. It occurs more frequently in males than in females (5:2) and more frequently on the left side. In our patient UPJO was in the lower moiety of a duplicated system, that is a rare type of this anomaly.

Iranian j m s

Uretero-vesical junction obstruction (UVJO)

UVJO is an obstruction at the level of the end of ureter where it enters the bladder. It is the result of an anatomic or a functional abnormality in the distal segment of the ureter (10). The usual cause for UVJO is a defect in the peristalsis of distal part of the ureter (an adynamic segment), that acts as obstruction and causes backward pressure to the kidney and finally presents with hydroureteronephrosis or megaureter. In our patient the UVJ was narrow, so that any catheter could not be passed for performing retrograde pyelography, but during exploration typical tapering of the common ureter was seen while the lower moiety ureter was thin and narrow. I think the lower moiety ureter was refluxing into the dilated upper moiety ureter. The patient underwent simultaneous pyeloplasty and anti-refluxing ureteroneocystostomy.

\section{Conclusions}

Congenital anomalies of the urinary tract are among the most anomalies and have developmental basis even before the metanephric kidney begins to develop. These anomalies may compromise the functional or conduit parts of the urinary tract. Multiple simultaneous congenital anomalies can be seen accidentally in an asymptomatic patient. In this reported case, renal ectopia; double collecting system, UPJO, UVJO, were seen and also suggested for reflux of lower moiety ureter into the upper moiety ureter.

\section{List of abbreviations}

UPJO: Ureter-pelvic Junction Obstruction

UVJO: Uretero-vesical Junction Obstruction

VUR: Vesico-ureteral Reflux

IVP: Intravenous Pyelography

DTPA: Dimercapto succinic Acid

\section{Declarations}

Ethics approval and consent to participate: Approval for the study was obtained from the Ethical Committee in biomedical Research in Yasuj University of Medical Sciences (the related code is: IR. Yums.rec.1399.184), and written informed consent was obtained from the study participant. 


\section{Consent for publication:}

Written informed consent for publication of the clinical details and clinical

images were obtained from the patient. A copy of the consent

form is available on reasonable request.

Availability of data and materials: The datasets used during the current study are available from the corresponding authoron reasonable request.

Competing interests: The authors declare that they have no competing interests.

Funding: Funding information is not applicable.

Authors' contributions: As I am the only author of the manuscript, the whole process of this manuscript including submission, have been provided by myself alone.

ACKNOWLEDGEMENTS: I thank Dr Zafar Masoumi (Department of radiology, Beheshti Teaching Hospital) for his constructive Suggestions and performing sonography and IVP.

Author information: SM Rabani is the author of this manuscript, and the corresponding author.

Affiliations: Department of Urology, Beheshti Teaching Hospital, Yasuj University of Medical Sciences, Yasuj, Iran

References

1 - Schedl A. Renal abnormalities and their developmental origin. Nat Rev Genet 2007; 8: 791-802

2 - Jain S. Overview of congenital anomalies of the kidney and urinary tract. In: Colvin RB, Chang AC (eds.). Diagnostic Pathology: Kidney Diseases, 2nd edn. Philadelphia: Elsevier, 2016, 828-831

3 - Dressler GR. Advances in early kidney specification, development and patterning. Development 2009; 136: $3863-3874$

4 - Hoshi M, Batourina E, Mendelsohn C et al. Novel mechanisms of early upper and lower urinary tract patterning regulated by RetY1015 docking tyrosine in mice. Development. 2012; 139: 2405-2415

5 - Renkema KY, Winyard PJ, Skovorodkin IN et al. Novel perspectives for investigating congenital anomalies of the kidney and urinary tract (CAKUT). Nephrol Dial Transplant

2011; 26: 3843-3851

6 - Chen F. Genetic and developmental basis for urinary tract obstruction. Pediatr Nephrol 2009; 24: $1621-1632$

7 - Whitten SM, Wilcox DT. Duplex systems. Prenat Diagn 2001; 21: 952-957

8 - Klein J, Gonzalez J, Miravete M et al. Congenital ureteropelvic junction obstruction: human disease and animal models. Int J Exp Pathol 2011; 92: 168-192

9 - Yiee JH, Johnson-Welch S, Baker LA et al. Histologic differences between extrinsic and intrinsic ureteropelvic junction obstruction. Urology 2010; 76: 181-184

10 - Rabani SM, Mousavizadeh A., The dilemma of ureterovesical junction obstruction, Nephro-urol mon. 2017; 9(5): e57201

Figure Legends 


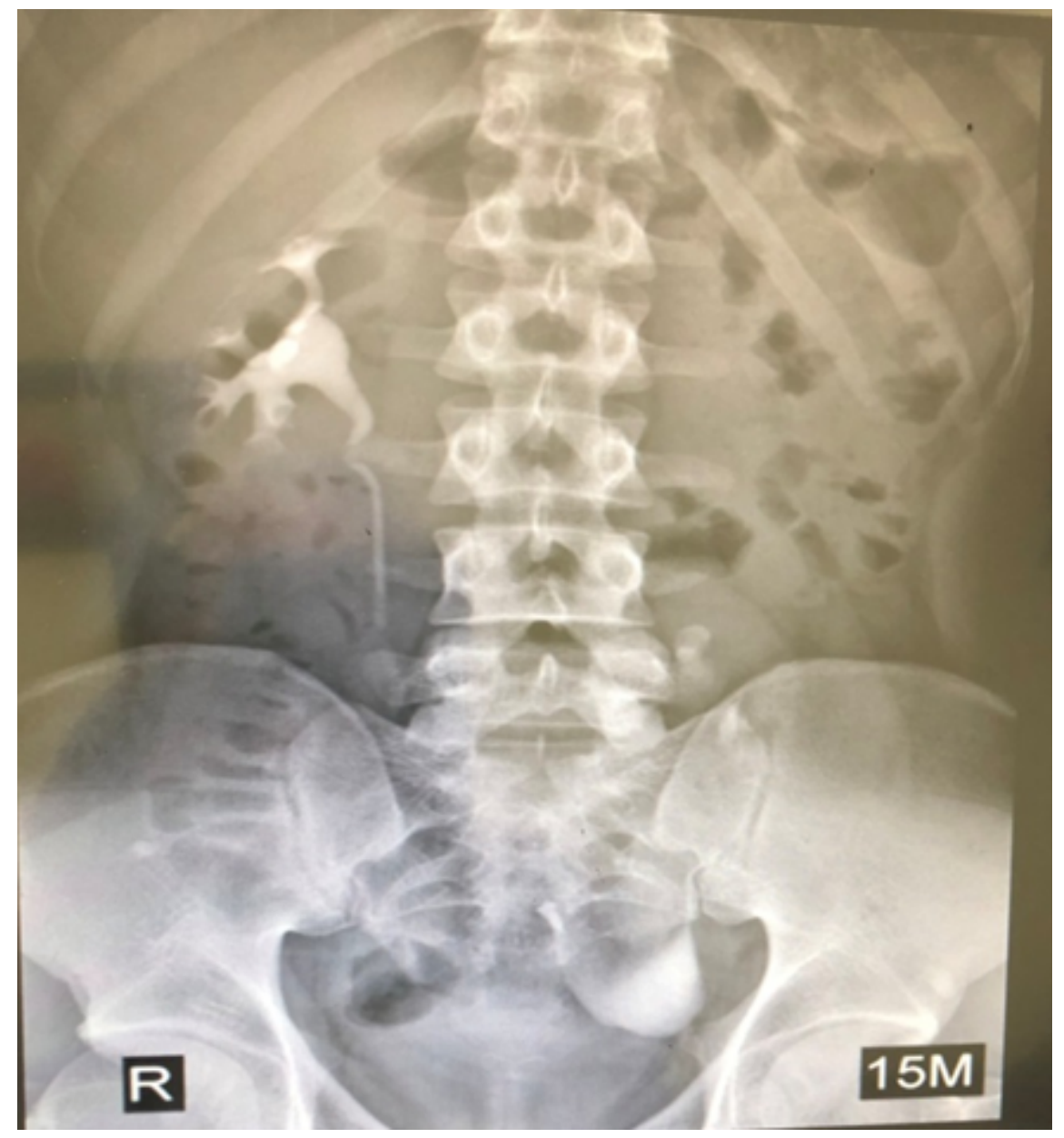

Figure 1: IVP suggestive for functional, ectopic, irregular, left kidney with hydroureteronephrosis. 


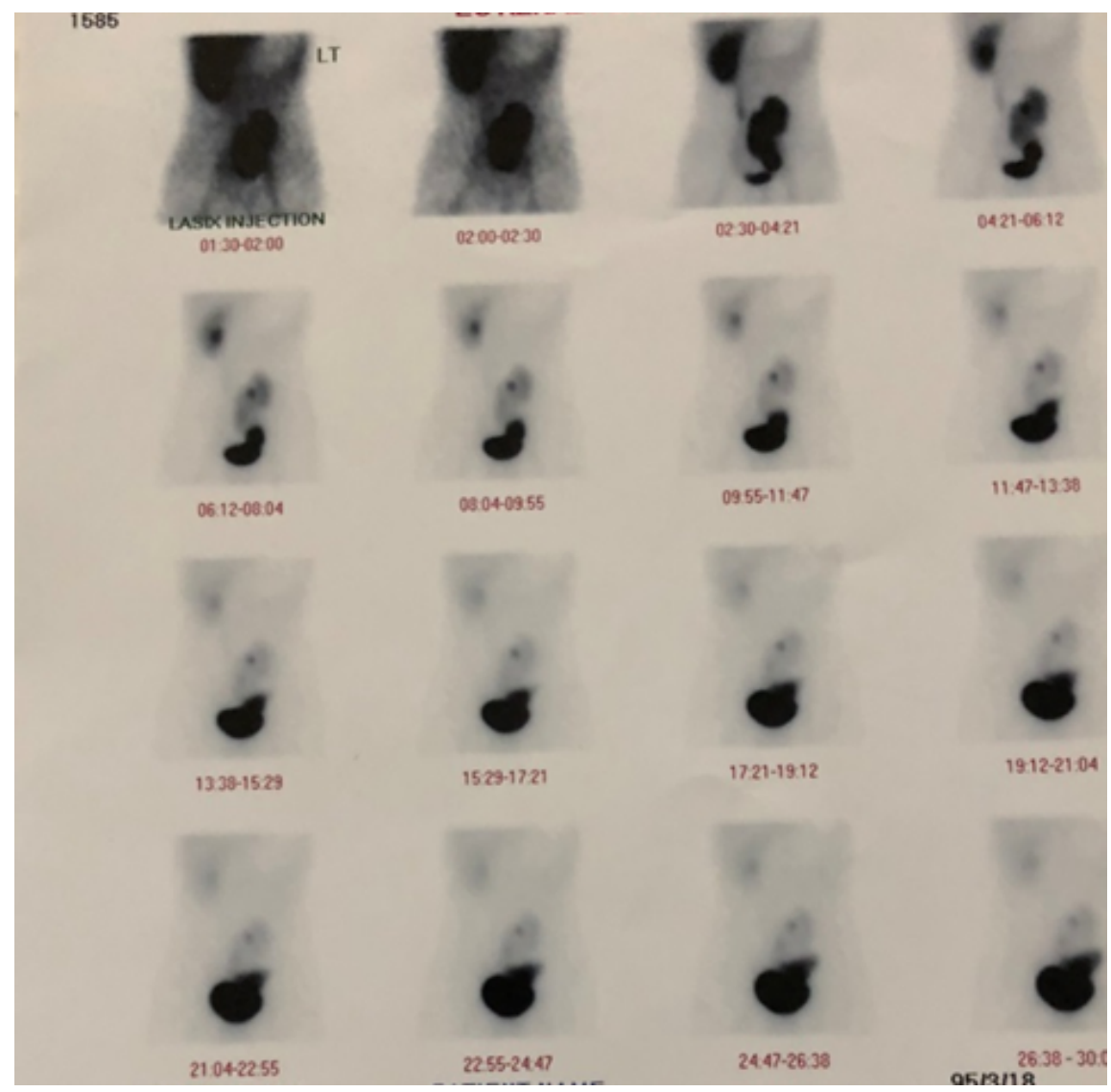

Figure 2: Renal DTPA scanning confirming the IVP findings. 